\title{
Regularized gradient-projection methods for finding the minimum-norm solution of equilibrium and the constrained convex minimization problem
}

\author{
Ming Tian ${ }^{a, b, *}$, Hui-Fang Zhang ${ }^{a}$ \\ ${ }^{a}$ College of Since, Civil Aviation University of China, Tianjin 300300, China. \\ ${ }^{b}$ Tianjin Key Laboratory for Advanced Signal Processing, Civil Aviation University of China, Tianjin 300300, China. \\ Communicated by Y. H. Yao
}

\begin{abstract}
The gradient-projection algorithm (GPA) is an effective method for solving the constrained convex minimization problem. Ordinarily, under some conditions, the minimization problem has more than one solution, so the regulation is used to find the minimum-norm solution of the minimization problem. In this article, we come up with a regularized gradient-projection algorithm to find a common element of the solution set of equilibrium and the solution set of the constrained convex minimization problem, which is the minimum-norm solution of equilibrium and the constrained convex minimization problem. Under some suitable conditions, we can obtain some strong convergence theorems. As an application, we apply our algorithm to solve the split feasibility problem and the constrained convex minimization problem in Hilbert spaces. (C) 2016 All rights reserved.
\end{abstract}

Keywords: Iterative method, equilibrium problem, constrained convex minimization problem, variational inequality, regularization, minimum-norm.

2010 MSC: 47H10, 54H25.

\section{Introduction}

Let $H$ be a real Hilbert space with inner product $\langle\cdot, \cdot\rangle$ and norm $\|\cdot\|$. Let $C$ be a subset of $H$, which is nonempty, closed and convex. Let $\mathbb{N}$ and $\mathbb{R}$ denote the sets of positive integers and real numbers, respectively.

\footnotetext{
${ }^{*}$ Corresponding author

Email addresses: tianming1963@126.com (Ming Tian), huifangzhang109@126.com (Hui-Fang Zhang)
} 
A nonlinear operator $T: C \rightarrow C$ is nonexpansive if $\|T x-T y\| \leq\|x-y\|$ for all $x, y \in C$. We use $F i x(T)$ to denote the fixed point of $T$. A nonlinear mapping $A: H \rightarrow H$ is monotone if $\langle x-y, A x-A y\rangle \geq 0$ for all $x, y \in H$. It can be easily seen that if $T$ is nonexpansive, then $I-T$ is monotone.

Firstly, consider the following equilibrium problem (EP), find $z \in C$ such that

$$
\varphi(z, y) \geq 0, \quad \forall y \in C,
$$

where $\varphi$ is a bifunction of $C \times C$ into $\mathbb{R}$. Assume the EP 1.1 is solvable, and denote the solution set of $\mathrm{EP}$ by $\operatorname{EP}(\varphi)$. We also know that the $\mathrm{EP}$ is equivalent to variational inequality problem, where a mapping $F: C \rightarrow H$, let $\varphi(x, y)=\langle F x, y-x\rangle$ for all $x, y \in C$. We can conclude that $E P(\varphi)$ is the solution set of the variational inequality.

As we all know that the equilibrium problem is widely used in many aspects such as physics, optimization and economics. Therefore, how to solve the equilibrium problem has became a hot problem. Many authors proposed different methods, we can see from the references [11, 12, 18, 20, 28].

Secondly, consider the constrained convex minimization problem as follows:

$$
\min _{x \in C} g(x),
$$

where $g: C \rightarrow \mathbb{R}$ is a real-valued convex function. Assume that the constrained convex minimization problem $(1.2)$ is solvable, let $U$ denote its solution set. A sequence $\left\{x_{n}\right\}$ generated by the following recursive formula:

$$
x_{n+1}=P_{C}\left(I-\lambda_{n} \nabla g\right) x_{n}, \quad \forall n \geq 0,
$$

is called the gradient-projection algorithm, where the parameters $\left\{\lambda_{n}\right\}$ are real positive numbers, and $P_{C}$ is the metric projection from $H$ onto $C$. In general, if the gradient $\nabla g$ is Lipschitz continuous and strongly monotone, then the sequence $\left\{x_{n}\right\}$ generated by 1.3 converges strongly to a minimizer of 1.2 , where the parameters $\left\{\lambda_{n}\right\}$ satisfy some suitable conditions. However, if the gradient $\nabla g$ is only to be inverse strongly monotone, the sequence $\left\{x_{n}\right\}$ generated by 1.3 converges weakly.

Recently, many authors not only combine the equilibrium problem with a fixed point problem [4, 14, 16, 17, 22, but also combine the constrained convex minimization problem with a fixed point problem [5]. So we can also composite iterative algorithms for finding a common solution of the equilibrium problem and the constrained convex minimization problem [23, 24].

Ordinarily, the gradient-projection method converges weakly, Xu [27] came up with two modifications of it, then obtained two strong convergence theorems. On the other hand, regularization can be used to find the minimum-norm solution of the minimization problem.

Thirdly, we consider the following regularized minimization problem:

$$
\min _{x \in C} g_{\beta}(x):=g(x)+\frac{\beta}{2}\|x\|^{2},
$$

where the regularization parameter $\beta$ is positive, $g$ is a convex function and gradient $\nabla g$ is $\frac{1}{L}$-ism. Then, a sequence $\left\{x_{n}\right\}$ generated by the following formula:

$$
x_{n+1}=P_{C}\left(I-\lambda \nabla g_{\beta_{n}}\right) x_{n}=P_{C}\left(I-\lambda\left(\nabla g+\beta_{n} I\right)\right) x_{n}, \quad \forall n \geq 0,
$$

where the regularization parameters $\beta_{n}$ is positive, $P_{C}$ is the metric projection from $H$ onto $C . \lambda$ is a positive number. Then, a sequence $\left\{x_{n}\right\}$ generated by (1.4) converges weakly.

In 2011, Ceng et al. 7] proposed a sequence $\left\{x_{n}\right\}$ generated by the following iterative algorithm:

$$
x_{n+1}=P_{C}\left[\theta_{n} r f\left(x_{n}\right)+\left(I-\theta_{n} \mu F\right) T_{n}\left(x_{n}\right)\right], \quad \forall n \geq 0,
$$

where $f: C \rightarrow H$ is an $l$-Lipschitzian mapping with a constant $l>0$, and $F: C \rightarrow H$ is a $k$-Lipschitzian and $\eta$-strongly monotone operator with constants $k, \eta>0 . \theta_{n}=\frac{2-\lambda_{n} L}{4}, P_{C}\left(I-\lambda_{n} \nabla g\right)=\theta_{n} I+\left(1-\theta_{n}\right) T_{n}$, for all $n \geq 0$. Then a sequence $\left\{x_{n}\right\}$ generated by (1.5) converges strongly to a minimizer of 1.2 . 
In 2014, Tian and Liu [24] firstly proposed implicit and explicit composite iterative algorithms for solving equilibrium and the constrained convex minimization problem.

The implicit iterative algorithm:

$$
\begin{cases}\varphi\left(u_{n}, y\right)+\frac{1}{r_{n}}\left\langle y-u_{n}, u_{n}-x_{n}\right\rangle \geq 0, & \forall y \in C, \\ x_{n}=\alpha_{n} f\left(x_{n}\right)+\left(1-\alpha_{n}\right) T_{n} u_{n}, & \forall n \in \mathbb{N} .\end{cases}
$$

The explicit iterative algorithm:

$$
\begin{cases}\varphi\left(u_{n}, y\right)+\frac{1}{r_{n}}\left\langle y-u_{n}, u_{n}-x_{n}\right\rangle \geq 0, & \forall y \in C \\ x_{n+1}=\alpha_{n} f\left(x_{n}\right)+\left(1-\alpha_{n}\right) T_{n} u_{n}, & \forall n \in \mathbb{N}\end{cases}
$$

where $u_{n}=Q_{r_{n}} x_{n}, P_{C}\left(I-\lambda_{n} \nabla g\right)=s_{n} I+\left(1-s_{n}\right) T_{n}, s_{n}=\frac{2-\lambda_{n} L}{4},\left\{r_{n}\right\},\left\{\alpha_{n}\right\},\left\{\lambda_{n}\right\}$ satisfy some suitable conditions, then a sequence $\left\{x_{n}\right\}$ generated by (1.6) or (1.7) converges strongly to a point $q \in U \cap E P(\varphi)$, which solves the variational inequality $\langle(I-f) q, p-q\rangle \geq 0$, for all $p \in U \cap E P(\varphi)$.

Then, in 2014, Lin [30] proposed the following iterative algorithm, for $x_{1} \in C$ :

$$
x_{n+1}=J_{\rho}\left(I-\rho\left(F+\beta_{n} I\right)\right) T_{r} x_{n}, \quad \forall n>0,
$$

where $J_{\rho}, T_{r}$ are the resovent of maximal monotone mapping $B, G, \rho$ is a constant where $0<\rho<\frac{2}{2+L}$, $\beta_{n} \in(0,1)$, and $F$ is $\frac{1}{L}$-ism. Then the sequence $\left\{x_{n}\right\}$ generated by 1.8 converges strongly to $\bar{x}$, where $\bar{x}=P_{(F+B)^{-1}(0) \cap G^{-1(0)}}(0)$.

From the article of Lin, we obtain a new condition of parameter $\rho, 0<\rho<\frac{2}{2+L}$, which is used widely in our article. By using this new condition, we obtain some new strong convergence theorems.

In this article, motivated inspired by Tian, Liu and Lin, [24, 30] we come up with a new iterative algorithm:

$$
\begin{cases}\varphi\left(u_{n}, y\right)+\frac{1}{r_{n}}\left\langle y-u_{n}, u_{n}-x_{n}\right\rangle \geq 0, & \forall y \in C, \\ x_{n+1}=P_{C}\left(I-\lambda\left(\nabla g+\beta_{n} I\right) u_{n},\right. & \forall n \in \mathbb{N},\end{cases}
$$

for finding an element of $U \cap E P(\varphi)$, where $\varphi: C \times C \rightarrow \mathbb{R}, P_{C}$ is the self-mapping on $C$. Under appropriate conditions, we can prove that the sequence $\left\{x_{n}\right\}$ generated by $(1.9)$ converges strongly to a point $q \in U \cap \operatorname{EP}(\varphi)$, where $q=P_{U \cap E P(\varphi)}(0)$ is the minimum-norm solution of equilibrium and the constrained convex minimization problem.

Finally, we give concrete examples and the numerical results to illustrate the practical value of our algorithm in the last section.

\section{Preliminaries}

In this part, we also introduce some lemmas and some properties that be used in the rest part. Throughout this paper, let $H$ be a real Hilbert space, and $C$ be a nonempty, closed, and convex subset of $H$. We also use the sign ${ }^{\prime} \rightarrow^{\prime}$ to denote that the sequence $\left\{x_{n}\right\}$ converges weakly to a point $x \in C$, and we use the sign ' $\rightarrow$ to denote that the sequence $\left\{x_{n}\right\}$ converges strongly to a point $x \in C$, and we use $F(T)$ to denote the fixed point of $T$.

Solving the equilibrium problem is not an easy thing, we should assume that $\varphi: C \times C \rightarrow \mathbb{R}$ satisfies the following conditions:

(A1) $\varphi(x, x)=0$ for all $x \in C$;

(A2) $\varphi$ is monotone, that is to say, $\varphi(x, y)+\varphi(y, x) \leq 0$ for all $x, y \in C$;

(A3) for each $x, y, z \in C, \lim _{t \rightarrow 0} \varphi(t z+(1-t) x, y) \leq \varphi(x, y)$;

(A4) for each $x \in C, y \mapsto \phi(x, y)$ is convex and lower semicontinuous.

Then, the equilibrium problem can be transformed as the fixed point problem. The following lemma plays an important role in solving equilibrium problem. 
Lemma 2.1 ([1, 10]). Let $\varphi$ be a bifunction of $C \times C$ into $\mathbb{R}$ satisfying (A1)-(A4). Then for any $r>0$ and $x \in H$, there exists $z \in C$ such that

$$
\varphi(z, y)+\frac{1}{r}\langle y-z, z-x\rangle \geq 0, \quad \forall y \in C .
$$

Then we define a mapping $Q_{r}: H \rightarrow C$ as follows:

$$
Q_{r}(x)=\left\{z \in C: \varphi(z, y)+\frac{1}{r}\langle y-z, z-x\rangle \geq 0, \quad \forall y \in C\right\} .
$$

Then, the following hold:

(1) $Q_{r}$ is single-valued;

(2) $Q_{r}$ is a firmly nonexpansive mapping, when for all $x, y \in H$,

$$
\left\|Q_{r} x-Q_{r} y\right\|^{2} \leq\left\langle Q_{r} x-Q_{r} y, x-y\right\rangle ;
$$

(3) $F i x\left(Q_{r}\right)=E P(\varphi)$;

(4) $\operatorname{EP}(\varphi)$ is closed and convex.

Takahashi gave the following lemma about equilibrium problem.

Lemma 2.2 ([21]). Let $H$ be a Hilbert space and $C$ be a nonempty, closed, and convex subset of $H$. Let $\varphi: C \times C \rightarrow \mathbb{R}$ satisfying (A1)-(A4). Let $A_{\varphi}$ be a set-valued mapping of $H$ into itself defined by

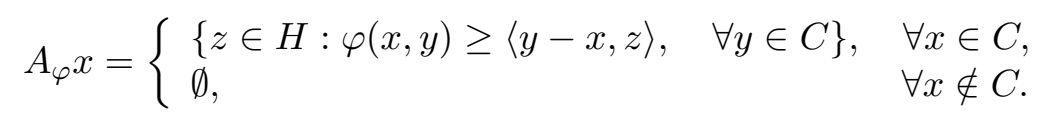

Then, $\operatorname{EP}(\varphi)=A_{\varphi}^{-1} 0$ and $A_{\varphi}$ is a maximal monotone operator with $\operatorname{dom}_{\varphi} \subset C$. Furthermore, for any $x \in H$ and $r>0$, the resolvent $Q_{r}$ of $\varphi$ coincides with the resolvent of $A_{\varphi}$, that is,

$$
Q_{r} x=\left(I+r A_{\varphi}\right)^{-1} x .
$$

Lemma $2.3([15])$. Let $H$ be a Hilbert space and $C$ be a nonempty closed convex subset of $H$. Let $i_{C}$ be the indicator function of $C$, then $i_{C}$ is a proper lower semicontinuous convex function on $H$ and the subdifferential $\partial i_{C}$ of $i_{C}$ is a maximal monotone operator. Define $Q_{\lambda} x=\left(I+\lambda \partial i_{C}\right)^{-1} x$, for all $x \in H$. We see that for any $x \in H$ and $u \in C$,

$$
u=Q_{\lambda} x \Longleftrightarrow u=P_{C} x .
$$

Then, we introduce some definitions and properties of the operators which are well-known in the literatures.

Definition $2.4([25])$. A mapping $T: H \rightarrow H$ is said to be an averaged mapping if it can be written as the average of the identity $I$ and a nonexpansive mapping, that is,

$$
T=(1-\alpha) I+\alpha S,
$$

where $\alpha \in(0,1)$ and $S: H \rightarrow H$ is nonexpansive. Then $T$ is called $\alpha$-averaged.

Definition $2.5([2])$. A nonlinear operator $B$ whose domain $D(B) \subseteq H$ and range $\mathbb{R}(B) \subseteq H$ is said to be:

(1) monotone if

$$
\langle x-y, B x-B y\rangle \geq 0, \quad \forall x, y \in D(B)
$$

(2) $\beta$-strongly monotone if there exists $\beta>0$ such that

$$
\langle x-y, B x-B y\rangle \geq \beta\|x-y\|^{2}, \quad \forall x, y \in D(B) ;
$$

(3) $v$-inverse strongly monotone $(v$-ism) if there exists $v>0$ such that

$$
\langle x-y, B x-B y\rangle \geq v\|B x-B y\|^{2}, \quad \forall x, y \in D(B) .
$$


Proposition 2.6 ([3]). Let $T: H \rightarrow H$ be an operator from $H$ to itself.

(1) $T$ is nonexpansive if and only if the complement $I-T$ is $\frac{1}{2}$-ism.

(2) If $T$ is $v$-ism, then for $r>0, r T$ is $\frac{v}{r}$-ism.

(3) $T$ is averaged if and only if the complement $I-T$ is $v$-ism, for some $v>\frac{1}{2}$. Indeed, for $\alpha \in(0,1), T$ is $\alpha$-averaged if and only if $I-T$ is $\frac{1}{2 \alpha}$-ism.

Recall that $P_{C}$ is the metric projection from $H$ into $C$, then for each point $x \in H$, the unique point $P_{C} \in C$ satisfies the property:

$$
\left\|x-P_{C} x\right\|=\inf _{y \in C}\|x-y\|=: d(x, C) .
$$

$P_{C}$ has the following characteristics.

Lemma 2.7 ([19]). For a given $x \in H$ :

(1) $z=P_{C} x$ if and only if $\langle x-z, z-y\rangle \geq 0, \quad \forall y \in C$;

(2) $z=P_{C} x$ if and only if $\|x-z\|^{2} \leq\|x-y\|^{2}-\|y-z\|^{2}, \quad \forall y \in C$;

(3) $\left\langle P_{C} x-P_{C} y, x-y\right\rangle \geq\left\|P_{C} x-P_{C} y\right\|^{2}, \quad \forall x, y \in H$.

From (3), we can derive that $P_{C}$ is nonexpansive and monotone. The inequality in the following lemma is always used in the process of proof.

Lemma $2.8([9])$. In an inner product space $X$,

$$
\|x+y\|^{2} \leq\|x\|^{2}+2\langle y, x+y\rangle, \quad \forall x, y \in X .
$$

The so-called demiclosedness principle for nonexpansive mapping and the last lemma are often used in the process of proof.

Lemma 2.9 ([13]). Let $T: C \rightarrow C$ be a nonexpansive mapping with $F(T) \neq \emptyset$. If $\left\{x_{n}\right\}$ is a sequence in $C$ weekly converging to $x$ and if $\left\{(I-T) x_{n}\right\}$ converges strongly to $y$, then $(I-T) x=y$. In particular, if $y=0$, then $x \in F(T)$.

Lemma $2.10([26])$. Let $\left\{a_{n}\right\}$ be a sequence of nonnegative real numbers such that

$$
a_{n+1} \leq\left(1-\alpha_{n}\right) a_{n}+\alpha_{n} \delta_{n}, \quad n \geq 0,
$$

where $\left\{\alpha_{n}\right\}_{n=0}^{\infty}$ and $\left\{\delta_{n}\right\}_{n=0}^{\infty}$ are sequences of real numbers in $(0,1)$ such that

(1) $\sum_{n=0}^{\infty} \alpha_{n}=\infty$;

(2) $\limsup _{n \rightarrow \infty} \delta_{n} \leq 0$ or $\sum_{n=0}^{\infty} \alpha_{n}\left|\delta_{n}\right|<\infty$.

Then $\lim _{n \rightarrow \infty} a_{n}=0$.

\section{Main results}

In this paper, we always assume that $g: C \rightarrow \mathbb{R}$ is real-valued convex function and the gradient $\nabla g$ is $\frac{1}{L}$-ism, which implies that $\lambda \nabla g$ is $\frac{1}{\lambda L}$-ism.

Suppose that the minimization problem 1.2 is consistent, and let $U$ denote solution set. Let $\left\{Q_{r_{n}}\right\}$ be a sequence of mappings defined as in Lemma 2.1. Consider the following mapping $G_{n}$ on $C$ defined by

$$
G_{n} x=P_{C}\left(I-\lambda\left(\nabla g+\beta_{n} I\right)\right) Q_{r_{n}} x, \quad \forall x \in C, n \in \mathbb{N},
$$

where $\lambda \in\left(0, \frac{2}{L+2}\right), \beta_{n} \in(0,1)$. By Lemma 2.1, we have 


$$
\begin{aligned}
\left\|G_{n} x-G_{n} y\right\|^{2}= & \left\|P_{C}\left(I-\lambda\left(\nabla g+\beta_{n} I\right)\right) Q_{r_{n}} x-P_{C}\left(I-\lambda\left(\nabla g+\beta_{n} I\right)\right) Q_{r_{n}} y\right\|^{2} \\
\leq & \left\|\left(I-\lambda\left(\nabla g+\beta_{n} I\right)\right) x-\left(I-\lambda\left(\nabla g+\beta_{n} I\right)\right) y\right\|^{2} \\
= & \left(1-\lambda \beta_{n}\right)^{2}\|x-y\|^{2}+\lambda^{2}\|\nabla g(x)-\nabla g(y)\|^{2} \\
& -2 \lambda\left(1-\lambda \beta_{n}\right)\langle x-y, \nabla g(x)-\nabla g(y)\rangle \\
\leq & \left(1-\lambda \beta_{n}\right)^{2}\|x-y\|^{2}+\lambda^{2}\|\nabla g(x)-\nabla g(y)\|^{2} \\
& -\frac{2}{L} \lambda\left(1-\lambda \beta_{n}\right)\|\nabla g(x)-\nabla g(y)\|^{2} \\
\leq & \left(1-\lambda \beta_{n}\right)^{2}\|x-y\|^{2}-\lambda\left(\frac{2}{L}(1-\lambda)-\lambda\right)\|\nabla g(x)-\nabla g(y)\|^{2} \\
\leq & \left(1-\lambda \beta_{n}\right)^{2}\|x-y\|^{2} .
\end{aligned}
$$

Then we have

$$
\left\|G_{n} x-G_{n} y\right\| \leq\left(1-\lambda \beta_{n}\right)\|x-y\| .
$$

Since $0<1-\lambda \beta_{n}<1$, it follows that $G_{n}$ is a contraction. Therefore, by the Banach contraction principle, $G_{n}$ has a unique fixed point $x_{n}^{*} \in C$, such that

$$
x_{n}^{*}=P_{C}\left(I-\lambda\left(\nabla g+\beta_{n} I\right)\right) Q_{r_{n}} x_{n}^{*} .
$$

For simplicity, we will write $x_{n}$ for $x_{n}^{*}$ provided no confusion occurs. Next, we prove the convergence of $\left\{x_{n}\right\}$, while we claim the existence of the $q \in U \cap E P(\varphi)$, which solves the variational inequality

$$
\langle-q, p-q\rangle \leq 0, \quad \forall p \in U \cap E P(\varphi) .
$$

Equivalently, $q=P_{U \cap E P(\varphi)}(0)$.

Theorem 3.1. Let $C$ be a nonempty closed convex subset of a real Hilbert space $H$ and $\varphi$ be a bifunction $C \times C$ into $\mathbb{R}$ satisfying (A1)-(A4). Let $g: C \rightarrow \mathbb{R}$ be real-valued convex function and assume that the gradient $\nabla g$ is $\frac{1}{L}$-ism with $L>0$. Assume that $U \cap E P(\varphi) \neq \emptyset$. Let $\left\{x_{n}\right\}$ be a sequence generated by

$$
\begin{cases}\varphi\left(u_{n}, y\right)+\frac{1}{r_{n}}\left\langle y-u_{n}, u_{n}-x_{n}\right\rangle \geq 0, & \forall y \in C, \\ x_{n}=P_{C}\left(I-\lambda\left(\nabla g+\beta_{n} I\right)\right) u_{n}, & \forall n \in \mathbb{N},\end{cases}
$$

where $u_{n}=Q_{r_{n}} x_{n}, 0<\lambda<\frac{2}{2+L}$. Let $\left\{r_{n}\right\}$ and $\left\{\beta_{n}\right\}$ satisfy the following conditions:

(i) $\left\{r_{n}\right\} \subset(0, \infty), \liminf _{n \rightarrow \infty} r_{n}>0$;

(ii) $\left\{\beta_{n}\right\} \subset(0,1), \lim _{n \rightarrow \infty} \beta_{n}=0, \sum_{n=1}^{\infty} \beta_{n}=\infty$.

Then $\left\{x_{n}\right\}$ converges strongly to a point $q \in U \cap E P(\varphi)$, where $q=P_{U \cap E P(\varphi)}(0)$.

Proof. First, we claim that $\left\{x_{n}\right\}$ is bounded. Indeed, pick any $p \in U \cap E P(\varphi)$, since $u_{n}=Q_{r_{n}} x_{n}$, and $p=Q_{r_{n}} p$, then we know that for any $n \in \mathbb{N}$,

$$
\left\|u_{n}-p\right\|=\left\|Q_{r_{n}} x_{n}-Q_{r_{n}} p\right\| \leq\left\|x_{n}-p\right\| .
$$

Thus, we derive that

$$
\begin{aligned}
\left\|x_{n}-p\right\|= & \left\|P_{C}\left(I-\lambda\left(\nabla g+\beta_{n} I\right)\right) Q_{r_{n}} x_{n}-P_{C}(I-\lambda \nabla g) Q_{r_{n}} p\right\| \\
\leq & \left\|\left(I-\lambda\left(\nabla g+\beta_{n} I\right)\right) x_{n}-\left(I-\lambda\left(\nabla g+\beta_{n} I\right)\right) p\right\| \\
& +\left\|\left(I-\lambda\left(\nabla g+\beta_{n} I\right)\right) p-(I-\lambda \nabla g) p\right\| \\
\leq & \left(1-\lambda \beta_{n}\right)\left\|x_{n}-p\right\|+\lambda \beta_{n}\|p\| .
\end{aligned}
$$


Then we have

$$
\left\|x_{n}-p\right\| \leq\|p\|,
$$

and hence $\left\{x_{n}\right\}$ is bounded. From 3.2 we also derive that $\left\{u_{n}\right\}$ is bounded.

Next, we obtain that $\left\|x_{n}-u_{n}\right\| \rightarrow 0$. Indeed, for any $p \in U \cap E P(\varphi)$, by Lemma 2.1, we have

$$
\begin{aligned}
\left\|u_{n}-p\right\|^{2} & =\left\|Q_{r_{n}} x_{n}-Q_{r_{n}} p\right\|^{2} \\
& \leq\left\langle x_{n}-p, u_{n}-p\right\rangle \\
& =\frac{1}{2}\left(\left\|x_{n}-p\right\|^{2}+\left\|u_{n}-p\right\|^{2}-\left\|u_{n}-x_{n}\right\|^{2}\right) .
\end{aligned}
$$

This implies that

$$
\left\|u_{n}-p\right\|^{2} \leq\left\|x_{n}-p\right\|^{2}-\left\|u_{n}-x_{n}\right\|^{2} .
$$

Then from (3.3) we derive that

$$
\begin{aligned}
\left\|x_{n}-p\right\|^{2}= & \left\|P_{C}\left(I-\lambda\left(\nabla g+\beta_{n} I\right)\right) u_{n}-P_{C}(I-\lambda \nabla g) p\right\|^{2} \\
= & \| P_{C}\left(I-\lambda\left(\nabla g+\beta_{n} I\right)\right) u_{n}-P_{C}\left(I-\lambda\left(\nabla g+\beta_{n} I\right)\right) p \\
& +P_{C}\left(I-\lambda\left(\nabla g+\beta_{n} I\right)\right) p-P_{C}(I-\lambda \nabla g) p \|^{2} \\
\leq & \left\|P_{C}\left(I-\lambda\left(\nabla g+\beta_{n} I\right)\right) u_{n}-P_{C}\left(I-\lambda\left(\nabla g+\beta_{n} I\right)\right) p\right\|^{2} \\
& +2\left\langle P_{C}\left(I-\lambda\left(\nabla g+\beta_{n} I\right)\right) p-P_{C}(I-\lambda \nabla g) p, x_{n}-p\right\rangle \\
\leq & \left(1-\lambda \beta_{n}\right)^{2}\left\|u_{n}-p\right\|^{2}+2 \lambda \beta_{n}\|p\| \cdot\left\|x_{n}-p\right\| \\
\leq & \left\|u_{n}-p\right\|^{2}+2 \lambda \beta_{n}\|p\| \cdot\left\|x_{n}-p\right\| \\
\leq & \left\|x_{n}-p\right\|^{2}-\left\|u_{n}-x_{n}\right\|^{2}+2 \lambda \beta_{n}\|p\| \cdot\left\|x_{n}-p\right\| .
\end{aligned}
$$

Since $\beta_{n} \rightarrow 0$ as $n \rightarrow \infty$, it follows that

$$
\lim _{n \rightarrow \infty}\left\|x_{n}-u_{n}\right\|=0, \text { as } n \rightarrow \infty .
$$

Then we show that $\left\|P_{C}(I-\lambda \nabla g) u_{n}-u_{n}\right\| \rightarrow 0$ as $n \rightarrow \infty$.

$$
\begin{aligned}
\left\|P_{C}(I-\lambda \nabla g) u_{n}-u_{n}\right\|= & \| P_{C}(I-\lambda \nabla g) u_{n}-P_{C}\left(I-\lambda\left(\nabla g+\beta_{n} I\right)\right) u_{n} \\
& +P_{C}\left(I-\lambda\left(\nabla g+\beta_{n} I\right)\right) u_{n}-u_{n} \| \\
\leq & \left\|P_{C}(I-\lambda \nabla g) u_{n}-P_{C}\left(I-\lambda\left(\nabla g+\beta_{n} I\right)\right) u_{n}\right\| \\
& +\left\|P_{C}\left(I-\lambda\left(\nabla g+\beta_{n} I\right)\right) u_{n}-u_{n}\right\| \\
\leq & \lambda \beta_{n}\left\|u_{n}\right\|+\left\|x_{n}-u_{n}\right\| .
\end{aligned}
$$

Since $\beta_{n} \rightarrow 0$ and $\left\|x_{n}-u_{n}\right\| \rightarrow 0$, we obtain that

$$
\left\|P_{C}(I-\lambda \nabla g) u_{n}-u_{n}\right\| \rightarrow 0, \text { as } n \rightarrow \infty .
$$

Since $\nabla g$ is $\frac{1}{L}$-ism, $P_{C}(I-\lambda \nabla g)$ is a nonexpansive self-mapping on $C$. As a matter of fact, we have for each $x, y \in C$

$$
\begin{aligned}
\left\|P_{C}(I-\lambda \nabla g) x-P_{C}(I-\lambda \nabla g) y\right\|^{2} & \leq\|(I-\lambda \nabla g) x-(I-\lambda \nabla g) y\|^{2} \\
& =\|x-y-\lambda(\nabla g(x)-\nabla g(y))\|^{2} \\
& =\|x-y\|^{2}-2 \lambda\langle x-y, \nabla g(x)-\nabla g(y)\rangle+\lambda^{2}\|\nabla g(x)-\nabla g(y)\|^{2} \\
& \leq\|x-y\|^{2}-\lambda\left(\frac{2}{L}-\lambda\right)\|\nabla g(x)-\nabla g(y)\|^{2} \\
& \leq\|x-y\|^{2} .
\end{aligned}
$$


Consider a subsequence $\left\{u_{n_{i}}\right\}$ of $\left\{u_{n}\right\}$. Since $\left\{u_{n_{i}}\right\}$ is bounded, there exists a subsequence $\left\{u_{n_{i_{j}}}\right\}$ of $\left\{u_{n_{i}}\right\}$ which converges weakly to $q$. Next, we show that $q \in U \cap E P(\varphi)$. Without loss of generality, we can assume that $u_{n_{i}} \rightarrow q$. Then by Lemma 2.9 , we obtain

$$
q=P_{C}(I-\lambda \nabla g) q .
$$

This shows that $q \in U$. Next, we show that $q \in E P(\varphi)$. Since $u_{n}=Q_{r_{n}} x_{n}$, for any $y \in C$, we obtain

$$
\varphi\left(u_{n}, y\right)+\frac{1}{r_{n}}\left\langle y-u_{n}, u_{n}-x_{n}\right\rangle \geq 0 .
$$

From (A2), we have

$$
\frac{1}{r_{n}}\left\langle y-u_{n}, u_{n}-x_{n}\right\rangle \geq \varphi\left(y, u_{n}\right)
$$

Replacing $n$ by $n_{i}$, we have

$$
\left\langle y-u_{n_{i}}, \frac{u_{n_{i}}-x_{n_{i}}}{r_{n_{i}}}\right\rangle \geq \varphi\left(y, u_{n_{i}}\right) .
$$

Since $\frac{u_{n_{i}}-x_{n_{i}}}{r_{n_{i}}} \rightarrow 0$ and $u_{n_{i}} \rightarrow q$, it follows from (A4) that $0 \geq \varphi(y, q)$, for all $y \in C$. Let

$$
z_{t}=t y+(1-t) q, \quad \forall t \in(0,1), \quad y \in C,
$$

then, we have $z_{t} \in C$ and hence $\varphi\left(z_{t}, q\right) \leq 0$. Thus, from (A1) and (A4), we have

$$
0=\varphi\left(z_{t}, z_{t}\right) \leq t \varphi\left(z_{t}, y\right)+(1-t) \varphi\left(z_{t}, q\right) \leq t \varphi\left(z_{t}, y\right)
$$

hence $0 \leq \varphi\left(z_{t}, y\right)$. From (A3), we have $0 \leq \varphi(q, y)$ for all $y \in C$, and so $q \in E P(\varphi)$. Therefore, $q \in U \cap \operatorname{EP}(\varphi)$. On the other hand, we note that

$$
x_{n}-q=P_{C}\left(I-\lambda\left(\nabla g+\beta_{n} I\right)\right) Q_{r_{n}} x_{n}-P_{C}(I-\lambda \nabla g) Q_{r_{n}} q .
$$

Hence, we obtain

$$
\begin{aligned}
\left\|x_{n}-q\right\|^{2}= & \left\|P_{C}\left(I-\lambda\left(\nabla g+\beta_{n} I\right)\right) Q_{r_{n}} x_{n}-P_{C}(I-\lambda \nabla g) Q_{r_{n}} q\right\|^{2} \\
\leq & \left\langle\left(I-\lambda\left(\nabla g+\beta_{n} I\right)\right) x_{n}-(I-\lambda \nabla g) q, x_{n}-q\right\rangle \\
= & \left\langle\left(I-\lambda\left(\nabla g+\beta_{n} I\right)\right) x_{n}-\left(I-\lambda\left(\nabla g+\beta_{n} I\right)\right) q, x_{n}-q\right\rangle \\
& +\left\langle-\lambda \beta_{n} q, x_{n}-q\right\rangle \\
\leq & \left(1-\lambda \beta_{n}\right)\left\|x_{n}-q\right\|^{2}+\lambda \beta_{n}\left\langle-q, x_{n}-q\right\rangle .
\end{aligned}
$$

It follows that

$$
\left\|x_{n}-q\right\|^{2} \leq\left\langle-q, x_{n}-q\right\rangle
$$

In particular,

$$
\left\|x_{n_{i}}-q\right\|^{2} \leq\left\langle-q, x_{n_{i}}-q\right\rangle .
$$

Since $\left\|x_{n}-u_{n}\right\| \rightarrow 0, u_{n_{i}} \rightarrow q$, we have that $x_{n_{i}} \rightarrow q$. Then we can derive that $x_{n_{i}} \rightarrow q$ as $i \rightarrow \infty$.

Next, we show that $q$ solves the variational inequality (3.1). Let $\widetilde{x}$ be the minimum-norm solution of $U \cap \operatorname{EP}(\varphi)$. That is, $\widetilde{x}=P_{U \cap E P(\varphi)}(0)$. Since $\left\{x_{n}\right\}$ is bounded, there exists a subsequence $\left\{x_{n_{i}}\right\}$ of $\left\{x_{n}\right\}$ such that $x_{n_{i}} \rightarrow z$. As the above proof, we know that $x_{n_{i}} \rightarrow z, z \in U \cap E P(\varphi)$. Then, we derive that

$$
\begin{aligned}
\left\|x_{n}-\widetilde{x}\right\|^{2} & =\left\|P_{C}\left(I-\lambda\left(\nabla g+\beta_{n} I\right)\right) Q_{r_{n}} x_{n}-P_{C}(I-\lambda \nabla g) Q_{r_{n}} \widetilde{x}\right\|^{2} \\
& \leq\left\langle\left(I-\lambda\left(\nabla g+\beta_{n} I\right)\right) x_{n}-(I-\lambda \nabla g) \widetilde{x}, x_{n}-\widetilde{x}\right\rangle \\
& =\left\langle\left(I-\lambda\left(\nabla g+\beta_{n} I\right)\right) x_{n}-\left(I-\lambda\left(\nabla g+\beta_{n} I\right)\right) \widetilde{x}, x_{n}-\widetilde{x}\right\rangle
\end{aligned}
$$




$$
\begin{aligned}
& +\left\langle-\lambda \beta_{n} \widetilde{x}, x_{n}-\widetilde{x}\right\rangle \\
\leq & \left(1-\lambda \beta_{n}\right)\left\|x_{n}-\widetilde{x}\right\|^{2}+\lambda \beta_{n}\left\langle-\widetilde{x}, x_{n}-\widetilde{x}\right\rangle .
\end{aligned}
$$

Thus,

$$
\left\|x_{n}-\widetilde{x}\right\|^{2} \leq\left\langle-\widetilde{x}, x_{n}-\widetilde{x}\right\rangle
$$

In particular,

$$
\left\|x_{n_{i}}-\widetilde{x}\right\|^{2} \leq\left\langle-\widetilde{x}, x_{n_{i}}-\widetilde{x}\right\rangle .
$$

Since $x_{n_{i}} \rightarrow z$, we have

$$
\|z-\widetilde{x}\|^{2} \leq\langle-\widetilde{x}, z-\widetilde{x}\rangle \leq 0 .
$$

Then, we have $z=\widetilde{x}$. From the arbitrariness of $z \in U \cap E P(\varphi)$, it follows that $q \in U \cap E P(\varphi)$ is a solution of the variational inequality (3.1). By the uniqueness of solution of the variational inequality (3.1), we conclude that $x_{n} \rightarrow q$ as $n \rightarrow \infty$, where $q=P_{U \cap E P(\varphi)}(0)$.

Theorem 3.2. Let $C$ be a nonempty closed convex subset of a real Hilbert space $H$ and $\varphi$ be a bifunction $C \times C$ into $\mathbb{R}$ satisfying (A1)-(A4). Let $g: C \rightarrow \mathbb{R}$ be real-valued convex function and assume that the gradient $\nabla g$ is $\frac{1}{L}$-ism with $L>0$. Assume that $U \cap E P(\varphi) \neq \emptyset$. Let $\left\{x_{n}\right\}$ be a sequence generated by $x_{1} \in C$ and

$$
\begin{cases}\varphi\left(u_{n}, y\right)+\frac{1}{r_{n}}\left\langle y-u_{n}, u_{n}-x_{n}\right\rangle \geq 0, & \forall y \in C, \\ x_{n+1}=P_{C}\left(I-\lambda\left(\nabla g+\beta_{n} I\right)\right) u_{n}, & \forall n \in \mathbb{N},\end{cases}
$$

where $u_{n}=Q_{r_{n}} x_{n}, 0<\lambda<\frac{2}{2+L}$. Let $\left\{r_{n}\right\}$ and $\left\{\beta_{n}\right\}$ satisfy the following conditions:

(i) $\left\{r_{n}\right\} \subset(0, \infty), \liminf _{n \rightarrow \infty} r_{n}>0, \sum_{n=1}^{\infty}\left|r_{n+1}-r_{n}\right|<\infty$;

(ii) $\left\{\beta_{n}\right\} \subset(0,1), \lim _{n \rightarrow \infty} \beta_{n}=0, \sum_{n=1}^{\infty} \beta_{n}=\infty, \sum_{n=1}^{\infty}\left|\beta_{n+1}-\beta_{n}\right|<\infty$.

Then $\left\{x_{n}\right\}$ strongly converges to a point $q \in U \cap E P(\varphi)$, where $q=P_{U \cap E P(\varphi)}(0)$.

Proof. First, we show that $\left\{x_{n}\right\}$ is bounded. Indeed, pick any $p \in U \cap E P(\varphi)$. Since $u_{n}=Q_{r_{n}} x_{n}$, and $p=Q_{r_{n}} p$, then we know that for any $n \in \mathbb{N}$,

$$
\left\|u_{n}-p\right\|=\left\|Q_{r_{n}} x_{n}-Q_{r_{n}} p\right\| \leq\left\|x_{n}-p\right\| .
$$

Thus, we derive that

$$
\begin{aligned}
\left\|x_{n+1}-p\right\|= & \left\|P_{C}\left(I-\lambda\left(\nabla g+\beta_{n} I\right)\right) Q_{r_{n}} x_{n}-P_{C}(I-\lambda \nabla g) Q_{r_{n}} p\right\| \\
\leq & \left\|\left(I-\lambda\left(\nabla g+\beta_{n} I\right)\right) x_{n}-\left(I-\lambda\left(\nabla g+\beta_{n} I\right)\right) p\right\| \\
& +\left\|\left(I-\lambda\left(\nabla g+\beta_{n} I\right)\right) p-(I-\lambda \nabla g) p\right\| \\
\leq & \left(1-\lambda \beta_{n}\right)\left\|x_{n}-p\right\|+\lambda \beta_{n}\|p\| \\
\leq & \max \left\{\left\|x_{n}-p\right\|,\|p\|\right\} .
\end{aligned}
$$

By the induction

$$
\left\|x_{n}-p\right\| \leq \max \left\{x_{1}-p\|,\| p \|\right\}
$$

and hence $\left\{x_{n}\right\}$ is bounded. From (3.4), we also derive that $\left\{u_{n}\right\}$ is bounded. Next, we show that $\| x_{n+1}-$ $x_{n} \| \rightarrow 0$.

$$
\begin{aligned}
\left\|x_{n+1}-x_{n}\right\|= & \left\|P_{C}\left(I-\lambda\left(\nabla g+\beta_{n} I\right)\right) u_{n}-P_{C}\left(I-\lambda\left(\nabla g+\beta_{n-1} I\right)\right) u_{n-1}\right\| \\
\leq & \left\|\left(I-\lambda\left(\nabla g+\beta_{n} I\right)\right) u_{n}-\left(I-\lambda\left(\nabla g+\beta_{n-1} I\right)\right) u_{n-1}\right\| \\
= & \|(I-\lambda \nabla g)\left(u_{n}-u_{n-1}\right) \\
& -\lambda \beta_{n}\left(u_{n}-u_{n-1}\right)-\lambda\left(\beta_{n}-\beta_{n-1}\right) u_{n-1} \|
\end{aligned}
$$




$$
\begin{aligned}
& \leq\left\|\left(I-\lambda\left(\nabla g+\beta_{n} I\right)\right)\left(u_{n}-u_{n-1}\right)\right\|+\lambda\left|\beta_{n}-\beta_{n-1}\right| \cdot\left\|u_{n-1}\right\| \\
& \leq\left(1-\lambda \beta_{n}\right)\left\|u_{n}-u_{n-1}\right\|+\lambda\left|\beta_{n}-\beta_{n-1}\right| \cdot\left\|u_{n-1}\right\| .
\end{aligned}
$$

From $u_{n+1}=Q_{r_{n+1}} x_{n+1}$, and $u_{n}=Q_{r_{n}} x_{n}$, we note that

$$
\varphi\left(u_{n+1}, y\right)+\frac{1}{r_{n+1}}\left\langle y-u_{n+1}, u_{n+1}-x_{n+1}\right\rangle \geq 0, \quad \forall y \in C,
$$

and

$$
\varphi\left(u_{n}, y\right)+\frac{1}{r_{n}}\left\langle y-u_{n}, u_{n}-x_{n}\right\rangle \geq 0, \quad \forall y \in C .
$$

By putting $y=u_{n}$ in (3.6) and $y=u_{n+1}$ in (3.7), we have

$$
\varphi\left(u_{n+1}, u_{n}\right)+\frac{1}{r_{n+1}}\left\langle u_{n}-u_{n+1}, u_{n+1}-x_{n+1}\right\rangle \geq 0, \quad \forall y \in C,
$$

and

$$
\varphi\left(u_{n}, u_{n+1}\right)+\frac{1}{r_{n}}\left\langle u_{n+1}-u_{n}, u_{n}-x_{n}\right\rangle \geq 0, \quad \forall y \in C .
$$

So, from (A2), we have

$$
\left\langle u_{n+1}-u_{n}, \frac{u_{n}-x_{n}}{r_{n}}-\frac{u_{n+1}-x_{n+1}}{r_{n+1}}\right\rangle \geq 0,
$$

and hence

$$
\left\langle u_{n+1}-u_{n}, u_{n}-u_{n+1}+u_{n+1}-x_{n}-\frac{r_{n}}{r_{n+1}}\left(u_{n+1}-x_{n+1}\right)\right\rangle \geq 0 .
$$

Since $\lim _{n \rightarrow \infty} r_{n}>0$, without loss of generality, let us assume that there exists a real number $a$, such that $r_{n}>a>0$ for all $n \in \mathbb{N}$. Thus, we have

$$
\begin{aligned}
\left\|u_{n+1}-u_{n}\right\|^{2} & \leq\left\langle u_{n+1}-u_{n}, x_{n+1}-x_{n}+\left(1-\frac{r_{n}}{r_{n+1}}\right)\left(u_{n+1}-x_{n+1}\right)\right\rangle \\
& \leq\left\|u_{n+1}-u_{n}\right\|\left\{\left\|x_{n+1}-x_{n}\right\|+\left|1-\frac{r_{n}}{r_{n+1}}\right| \cdot\left\|u_{n+1}-x_{n+1}\right\|\right\},
\end{aligned}
$$

thus

$$
\left\|u_{n+1}-u_{n}\right\| \leq\left\|x_{n+1}-x_{n}\right\|+\frac{1}{a}\left|r_{n+1}-r_{n}\right| M_{1},
$$

where $M_{1}=\sup \left\{\left\|u_{n}-x_{n}\right\|: n \in \mathbb{N}\right\}$. From (3.5) and 3.8), we obtain

$$
\begin{aligned}
\left\|x_{n+1}-x_{n}\right\| & \leq\left(1-\lambda \beta_{n}\right)\left(\left\|x_{n}-x_{n-1}\right\|+\frac{1}{a}\left|r_{n}-r_{n-1}\right| M_{1}\right)+\lambda\left|\beta_{n}-\beta_{n-1}\right| \cdot\left\|u_{n-1}\right\| \\
& \leq\left(1-\lambda \beta_{n}\right)\left\|x_{n}-x_{n-1}\right\|+\left(\left|r_{n}-r_{n-1}\right|+\left|\beta_{n}-\beta_{n-1}\right|\right) M_{2},
\end{aligned}
$$

where $M_{2}=\max \left\{\lambda\left\|u_{n-1}\right\|,\left(1-\lambda \beta_{n}\right) \frac{M_{1}}{a}\right\}$. Hence, by Lemma 2.10, we have

$$
\lim _{n \rightarrow \infty}\left\|x_{n+1}-x_{n}\right\|=0 .
$$

For any $p \in U \cap E P(\varphi)$, as in the proof of Theorem 3.1, we have

$$
\left\|u_{n}-p\right\|^{2} \leq\left\|x_{n}-p\right\|^{2}-\left\|u_{n}-x_{n}\right\|^{2} .
$$

Then from (3.9), we derive that

$$
\begin{aligned}
\left\|x_{n+1}-p\right\|^{2} & =\left\|P_{C}\left(I-\lambda\left(\nabla g+\beta_{n} I\right)\right) u_{n}-P_{C}(I-\lambda \nabla g) p\right\|^{2} \\
& =\| P_{C}\left(I-\lambda\left(\nabla g+\beta_{n} I\right)\right) u_{n}-P_{C}\left(I-\lambda\left(\nabla g+\beta_{n} I\right)\right) p
\end{aligned}
$$




$$
\begin{aligned}
& +P_{C}\left(I-\lambda\left(\nabla g+\beta_{n} I\right)\right) p-P_{C}(I-\lambda \nabla g) p \|^{2} \\
\leq & \left\|P_{C}\left(I-\lambda\left(\nabla g+\beta_{n} I\right)\right) u_{n}-P_{C}\left(I-\lambda\left(\nabla g+\beta_{n} I\right)\right) p\right\|^{2} \\
& +2\left\langle P_{C}\left(I-\lambda\left(\nabla g+\beta_{n} I\right)\right) p-P_{C}(I-\lambda \nabla g) p, x_{n+1}-p\right\rangle \\
\leq & \left(1-\lambda \beta_{n}\right)^{2}\left\|u_{n}-p\right\|^{2}+2 \lambda \beta_{n}\|p\| \cdot\left\|x_{n+1}-p\right\| \\
\leq & \left\|u_{n}-p\right\|^{2}+2 \lambda \beta_{n}\|p\| \cdot\left\|x_{n+1}-p\right\| \\
\leq & \left\|x_{n}-p\right\|^{2}-\left\|u_{n}-x_{n}\right\|^{2}+2 \lambda \beta_{n}\|p\| \cdot\left\|x_{n+1}-p\right\| .
\end{aligned}
$$

Then, we have that

$$
\begin{aligned}
\left\|u_{n}-x_{n}\right\|^{2} & \leq\left\|x_{n}-p\right\|^{2}-\left\|x_{n+1}-p\right\|^{2}+2 \lambda \beta_{n}\|p\| \cdot\left\|x_{n+1}-p\right\| \\
& \leq\left(\left\|x_{n}-p\right\|+\left\|x_{n+1}-p\right\|\right)\left\|x_{n+1}-x_{n}\right\|+2 \lambda \beta_{n}\|p\| \cdot\left\|x_{n+1}-p\right\| \\
& =\left\|x_{n+1}-x_{n}\right\| \cdot M_{3}+2 \lambda \beta_{n}\|p\| \cdot\left\|x_{n+1}-p\right\|,
\end{aligned}
$$

where $M_{3}=\sup \left\{\left\|x_{n}-p\right\|+\left\|x_{n+1}-p\right\|: n \in \mathbb{N}\right\}$. Since $\beta_{n} \rightarrow 0$ and $\left\|x_{n+1}-x_{n}\right\| \rightarrow 0$, we have

$$
\lim _{n \rightarrow \infty}\left\|x_{n}-u_{n}\right\|=0
$$

Then, we derive that

$$
\left\|x_{n+1}-u_{n}\right\|=\left\|x_{n+1}-x_{n}+x_{n}-u_{n}\right\| \leq\left\|x_{n+1}-x_{n}\right\|+\left\|x_{n}-u_{n}\right\| .
$$

So,

$$
\lim _{n \rightarrow \infty}\left\|x_{n+1}-u_{n}\right\|=0
$$

It follows that

$$
\left\|P_{C}\left(I-\lambda\left(\nabla g+\beta_{n} I\right)\right) u_{n}-u_{n}\right\| \rightarrow 0 .
$$

Then, we can know that

$$
\begin{aligned}
\left\|P_{C}(I-\lambda \nabla g) u_{n}-u_{n}\right\|= & \left\|P_{C}(I-\lambda \nabla g) u_{n}-P_{C}\left(I-\lambda\left(\nabla g+\beta_{n} I\right)\right) u_{n}\right\| \\
& +\left\|P_{C}\left(I-\lambda\left(\nabla g+\beta_{n} I\right)\right) u_{n}-u_{n}\right\| \\
\leq & \lambda \beta_{n}\left\|u_{n}\right\|+\left\|x_{n+1}-u_{n}\right\|,
\end{aligned}
$$

since $\beta_{n} \rightarrow 0$, and $\left\|x_{n+1}-u_{n}\right\| \rightarrow 0$, as $n \rightarrow \infty$, we obtain that

$$
\left\|P_{C}(I-\lambda \nabla g) u_{n}-u_{n}\right\| \rightarrow 0, \text { as } n \rightarrow \infty .
$$

Now, we show that

$$
\limsup _{n \rightarrow \infty}\left\langle-q, x_{n}-q\right\rangle \leq 0
$$

where $q=P_{U \cap E P(\varphi)}(0)$ is a unique solution of the variational inequality (3.1). Indeed, take a subsequence $\left\{x_{n_{j}}\right\}$ of $\left\{x_{n}\right\}$ such that

$$
\limsup _{n \rightarrow \infty}\left\langle-q, x_{n}-q\right\rangle=\lim _{j \rightarrow \infty}\left\langle-q, x_{n_{j}}-q\right\rangle .
$$

Since $\left\{x_{n}\right\}$ is bounded, without loss of generality, we may assume that $x_{n_{j}} \rightarrow z$. By the same argument as in the proof of Theorem 3.1, we have $z \in U \cap E P(\varphi)$. Since $q=P_{U \cap E P(\varphi)}(0)$, it follows that

$$
\limsup _{n \rightarrow \infty}\left\langle-q, x_{n}-q\right\rangle=\lim _{j \rightarrow \infty}\left\langle-q, x_{n_{j}}-q\right\rangle=\langle-q, z-q\rangle \leq 0 .
$$

From

$$
x_{n+1}-q=P_{C}\left(I-\lambda\left(\nabla g+\beta_{n} I\right)\right) u_{n}-q
$$




$$
=P_{C}\left(I-\lambda\left(\nabla g+\beta_{n} I\right)\right) Q_{r_{n}} x_{n}-P_{C}(I-\lambda \nabla g) Q_{r_{n}} q
$$

we have

$$
\begin{aligned}
\left\|x_{n+1}-q\right\|^{2}= & \left\|P_{C}\left(I-\lambda\left(\nabla g+\beta_{n} I\right)\right) Q_{r_{n}} x_{n}-P_{C}(I-\lambda \nabla g) Q_{r_{n}} q\right\|^{2} \\
\leq & \left\langle\left(I-\lambda\left(\nabla g+\beta_{n} I\right)\right) Q_{r_{n}} x_{n}-(I-\lambda \nabla g) Q_{r_{n}} q, x_{n+1}-q\right\rangle \\
\leq & \left\langle\left(I-\lambda\left(\nabla g+\beta_{n} I\right)\right) x_{n}-\left(I-\lambda\left(\nabla g+\beta_{n} I\right)\right) q, x_{n+1}-q\right\rangle \\
& +\lambda \beta_{n}\left\langle-q, x_{n+1}-q\right\rangle \\
\leq & \left(1-\lambda \beta_{n}\right)\left\|x_{n}-q\right\| \cdot\left\|x_{n+1}-q\right\|+\lambda \beta_{n}\left\langle-q, x_{n+1}-q\right\rangle \\
\leq & \frac{1-\lambda \beta_{n}}{2}\left\|x_{n}-q\right\|^{2}+\frac{1}{2}\left\|x_{n+1}-q\right\|^{2}+\lambda \beta_{n}\left\langle-q, x_{n+1}-q\right\rangle .
\end{aligned}
$$

It follows that

$$
\begin{aligned}
\left\|x_{n+1}-q\right\|^{2} & \leq\left(1-\lambda \beta_{n}\right)\left\|x_{n}-q\right\|^{2}+2 \lambda \beta_{n}\left\langle-q, x_{n+1}-q\right\rangle \\
& =\left(1-\lambda \beta_{n}\right)\left\|x_{n}-q\right\|^{2}+2 \lambda \beta_{n} \delta_{n}
\end{aligned}
$$

where $\delta_{n}=\left\langle-q, x_{n+1}-q\right\rangle$.

It is easy to see that $\lim _{n \rightarrow \infty} \lambda \beta_{n}=0, \sum_{n=1}^{\infty} \lambda \beta_{n}=\infty$, and $\limsup _{n \rightarrow \infty} \delta_{n} \leq 0$. Hence, by Lemma 2.10, the sequence $\left\{x_{n}\right\}$ converges strongly to $q$, where $q=P_{U \cap E P \varphi)}(0)$. This completes the proof.

Remark 3.3.

(i) Ordinarily, the regularization parameters $\beta_{n}$ is positive in (1.4), but in Theorem 3.1 and Theorem 3.2 , $0<\beta_{n}<1$.

(ii) $0<\lambda<\frac{2}{2+L}$, it is an important condition in Theorem 3.1 and Theorem 3.2 .

\section{Application}

In this part, we will illustrate the practical value of our algorithm in the split feasibility problem and the constrained convex minimization problem.

In 1994, Censor and Elfving [8] come up with the split feasibility problem. Many authors obtained some results on the split feasibility problem [29]. In this article, the SFP can be mathematically formulated as finding a point $x$ satisfying the following property:

$$
x \in C \text { and } A x \in Q,
$$

where $C$ and $Q$ are nonempty closed and convex subset of real Hilbert spaces $H_{1}$ and $H_{2}$, respectively and $A: H_{1} \rightarrow H_{2}$ is bounded linear operator.

$x^{*}$ is a solution of SFP if $x^{*} \in C$ and $A x^{*}-P_{Q} A x^{*}=0$. So, in order to find the solution of SFP, we should consider the constrained convex minimization problem:

$$
\min _{x \in C} g(x)=\min _{x \in C} \frac{1}{2}\left\|A x-P_{Q} A x\right\|^{2} .
$$

It is clear that if $x^{*}$ is the solution of SFP (4.1), that is, $x^{*}$ solves the minimization problem 4.2 and the minimum of 4.2 is 0 , then, the gradient of $g$ is $\nabla g$, where $\nabla g=A^{*}\left(I-P_{Q}\right) A$. We can calculate that $\nabla g$ is $\frac{1}{\|A\|^{2}}$-ism. So, by applying Theorem 3.2 , we can obtain the following theorem.

Theorem 4.1. Assume that the SFP 4.1) is consistent. Let $C$ be a nonempty closed convex subset of a real Hilbert space $H$ and $\varphi$ be a bifunction $C \times C$ into $\mathbb{R}$ satisfying (A1)-(A4). Let $g: C \rightarrow \mathbb{R}$ be realvalued convex function and assume that the gradient $\nabla g$ is $\frac{1}{\|A\|^{2}}$-ism, where $A: H_{1} \rightarrow H_{2}$ is bounded linear 
operator. Assume that $W \cap E P(\varphi) \neq \emptyset$, where $W$ denotes the solution set of SFP (4.1). Let $\left\{x_{n}\right\}$ be a sequence generated by $x_{1} \in C$ and

$$
\begin{cases}\varphi\left(u_{n}, y\right)+\frac{1}{r_{n}}\left\langle y-u_{n}, u_{n}-x_{n}\right\rangle \geq 0, & \forall y \in C, \\ x_{n+1}=P_{C}\left(I-\lambda\left(A^{*}\left(I-P_{Q}\right) A+\beta_{n} I\right)\right) u_{n}, & \forall n \in \mathbb{N}\end{cases}
$$

where $u_{n}=Q_{r_{n}} x_{n}, 0<\lambda<\frac{2}{2+L}$. Let $\left\{r_{n}\right\}$ and $\left\{\beta_{n}\right\}$ satisfy the following conditions:

(i) $\left\{r_{n}\right\} \subset(0, \infty), \liminf _{n \rightarrow \infty} r_{n}>0, \sum_{n=1}^{\infty}\left|r_{n+1}-r_{n}\right|<\infty$;

(ii) $\left\{\beta_{n}\right\} \subset(0,1), \lim _{n \rightarrow \infty} \beta_{n}=0, \sum_{n=1}^{\infty} \beta_{n}=\infty, \sum_{n=1}^{\infty}\left|\beta_{n+1}-\beta_{n}\right|<\infty$.

Then $\left\{x_{n}\right\}$ strongly converges to a point $q \in W \cap E P(\varphi)$, where $q=P_{W \cap E P(\varphi)}(0)$.

Proof. In this part, we only need to show that $\nabla g$ is $\frac{1}{\|A\|^{2}}$-ism, then the Theorem 4.1 can be obtained by Theorem 3.2 ,

$$
\nabla g=A^{*}\left(I-P_{Q}\right) A .
$$

Since $P_{Q}$ is firmly nonexpansive, so $P_{Q}$ is $\frac{1}{2}$-averaged mapping, then $I-P_{Q}$ is 1 -ism, for any $x, y \in C$, we derive that

$$
\begin{aligned}
\langle\nabla g(x)-\nabla g(y), x-y\rangle & =\left\langle A^{*}\left(I-P_{Q}\right) A x-A^{*}\left(I-P_{Q}\right) A y, x-y\right\rangle \\
& =\left\langle\left(I-P_{Q}\right) A x-\left(I-P_{Q}\right) A y, A x-A y\right\rangle \\
& \geq\left\|\left(I-P_{Q}\right) A x-\left(I-P_{Q}\right) A y\right\|^{2} \\
& =\frac{1}{\|A\|^{2}} \cdot\left\|A^{*}\left(\left(I-P_{Q}\right) A x-\left(I-P_{Q}\right) A y\right)\right\|^{2} \\
& =\frac{1}{\|A\|^{2}} \cdot\|\nabla g(x)-\nabla g(y)\|^{2} .
\end{aligned}
$$

So, $\nabla g$ is $\frac{1}{\|A\|^{2}}$-ism.

\section{Numerical results}

In this part, we use the algorithms in Theorem 4.1 and Theorem 3.2 to solve a system of linear equations and a constrained convex minimization problem.

First, we use the algorithm in Theorem 4.1 to calculate the $4 \times 4$ system of linear equations.

Example 5.1. Let $H_{1}=H_{2}=\mathbb{R}^{4}$. Take

$$
\begin{gathered}
A=\left(\begin{array}{cccc}
2 & 1 & -5 & 1 \\
1 & -3 & 0 & -6 \\
0 & 2 & -1 & 2 \\
1 & 4 & -7 & 6
\end{array}\right) \\
b=\left(\begin{array}{c}
8 \\
9 \\
-5 \\
0
\end{array}\right)
\end{gathered}
$$

Then the SFP can be formulated as the problem of finding a point $x^{*}$ with the property

$$
x^{*} \in C \text { and } A x^{*} \in Q,
$$


where $C=\mathbb{R}^{4}, Q=\{b\}$. That is, $x^{*}$ is the solution of the system of linear equations $A x=b$, and

$$
x^{*}=\left(\begin{array}{c}
3 \\
-4 \\
-1 \\
1
\end{array}\right) \text {. }
$$

Let $\varphi(z, y)=0$, for all $y \in C$. Take $P_{C}=I$, where $I$ denotes the $4 \times 4$ identity matrix. Consider the parameters $\beta_{n}=\frac{1}{n+1}$ for $n \geq 0, \lambda=\frac{1}{100}$. Then by Theorem 4.1 and Lemma 2.10, the sequence $\left\{x_{n}\right\}$ is generated by

$$
x_{n+1}=x_{n}-\frac{1}{100} A^{*} A x_{n}+\frac{1}{100} A^{*} b-\frac{1}{100(n+1)} x_{n} .
$$

As $n \rightarrow \infty$, we have $\left\{x_{n}\right\} \rightarrow x^{*}=(3,-4,-1,1)^{T}$.

\begin{tabular}{cccccc}
\hline$n$ & $x_{n}^{1}$ & $x_{n}^{2}$ & $x_{n}^{3}$ & $x_{n}^{4}$ & $E_{n}$ \\
\hline 0 & 1.0000 & 1.0000 & 1.0000 & 1.0000 & $5.74 \mathrm{E}+00$ \\
100 & 2.3080 & -1.7560 & -0.9960 & -0.3584 & $2.71 \mathrm{E}+00$ \\
500 & 2.7484 & -3.8529 & -1.0611 & 0.8762 & $2.95 \mathrm{E}-01$ \\
1000 & 2.9637 & -3.9930 & -1.0123 & 0.9888 & $4.05 \mathrm{E}-02$ \\
5000 & 2.9982 & -3.9988 & -1.0005 & 0.9990 & $2.50 \mathrm{E}-03$ \\
10000 & 2.9991 & -3.9994 & -1.0002 & 0.9995 & $1.20 \mathrm{E}-03$ \\
\hline
\end{tabular}

Table 1: Numerical results as regards Example 5.1 .

From Table1, we can easily see that with iterative number increasing $x_{n}$ approaches to the exact solution $x^{*}$ and the errors gradually approach to zero.

Second, we use the algorithm in Theorem 3.2 to solve the constrained convex minimization problem.

\begin{tabular}{ccc}
\hline$n$ & $x_{n}$ & $E_{n}$ \\
\hline 0 & 0.5000 & $5.00 \mathrm{E}-01$ \\
10 & 0.7377 & $2.62 \mathrm{E}-01$ \\
50 & 0.9407 & $5.93 \mathrm{E}-02$ \\
500 & 0.9945 & $5.50 \mathrm{E}-03$ \\
1000 & 0.9973 & $2.700 \mathrm{E}-03$ \\
5000 & 0.9995 & $5.44 \mathrm{E}-04$ \\
\hline
\end{tabular}

Table 2: Numerical results as regards Example 5.2

Example 5.2. Let $H=\mathbb{R}$ and $C=[0,2]$. Consider the problem 1.2$]$ and take function

$$
g(x)=\frac{-x}{e^{x}}, \quad \forall x \in C .
$$

The problem 1.2 can be written as

$$
\min _{x \in[0,2]} \frac{-x}{e^{x}}
$$

It can be seen that $\nabla g=\frac{x-1}{e^{x}}$, we can calculate that $\nabla g$ is $\frac{1}{2}$-ism, so, $L=2$, and $g(x)$ reaches the minimum at $x^{*}$, and $x^{*}=1$. Let $\varphi(z, y)=0$, for all $y \in C$. Take $P_{C}=I$, where $I$ denotes the unit function. Given the parameters $\beta_{n}=\frac{1}{n+1}$, for every $n \geq 0, \lambda=\frac{1}{4}$.

Then by Theorem 3.2 and Lemma 2.10 , the sequence $\left\{x_{n}\right\}$ is generated by

$$
x_{n+1}=x_{n}-\frac{1}{4}\left(\frac{x_{n}}{e^{x_{n}}}-\frac{1}{e^{x_{n}}}+\frac{x_{n}}{n+1}\right) .
$$


As $n \rightarrow \infty$, we have $\left\{x_{n}\right\} \rightarrow x^{*}$.

From Table 2, we easily know that by using the regularization method with iterative number increasing, $x_{n}$ approaches to $x^{*}$ and the errors gradually approach to zero.

From the two examples as above, we clearly can know about the practical value of our algorithms in application.

\section{Acknowledgment}

The first author is supported by the Foundation of Tianjin Key Laboratory For Advanced Signal Processing and is supported by Fundamental Research Funds for the Central Universities (GRANT:3122016L006).

\section{References}

[1] E. Blum, W. Oettli, From optimization and variational inequalities to equilibrium problems, Math. Student, 63 (1994), 123-145. 2.1

[2] H. Brézis, Opérateurs maximaux monotones et semi-groupes de contractions dans les espaces de Hilbert, (French) North-Holland Mathematics Studies, Notas de Matemática, North-Holland Publishing Co., Amsterdam-London, American Elsevier Publishing Co., Inc., New York, (1973). 2.5

[3] C. Byrne, A unified treatment of some iterative algorithms in signal processing and image reconstruction, Inverse Problems, 20 (2004), 103-120. 2.6

[4] L. C. Ceng, S. Al-Homidan, Q. H. Ansari, J. C. Yao, An iterative scheme for equilibrium problems and fixed point problems of strict pseudo-contraction mappings, J. Compt. Appl. Math., 223 (2009), 967-974. 1

[5] L. C. Ceng, Q. H. Ansari, C. F. Wen, Multi-step implicit iterative methods with regularization for minimization problems and fixed point problems, J. Inequal. Appl., 2013 (2013), 26 pages. 1

[6] L. C. Ceng, Q. H. Ansari, J. C. Yao, Extragradient-projection method for solving constrained convex minimization problems, Numer. Algebra Control Optim., 1 (2011), 341-359.

[7] L. C. Ceng, Q. H. Ansari, J. C. Yao, Some iterative methods for finding fixed points and for solving constrained convex minimization problems, Nonlinear Anal., 74 (2011), 5286-5302. 11, 1

[8] Y. Censor, T. Elfving, A multiprojection algorithm using Bregman projections in a product space, Numer. Algorithms, 8 (1994), 221-239. 4

[9] S.-S. Chang, Some problems and results in the study of nonlinear analysis, Proceedings of the Second World Congress of Nonlinear Analysts, Part 7 (Athens, 1996), Nonlinear Anal., 30 (1997), 4197-4208. 2.8

[10] P. L. Combettes, S. A. Hirstoaga, Equilibrium programming in Hilbert spaces, J. Nonlinear Convex Anal., 6 (2005), 117-136. 2.1

[11] S. D. Flam, A. S. Antipin, Equilibrium programming using proximal-like algorithms, Math. Programming, 78 (1997), 29-41. 1

[12] H. He, S. Liu, Y. J. Cho, An explicit method for systems of equilibrium problems and fixed points of infinite family of nonexpansive mappings, J. Comput. Appl. Math., 235 (2011), 4128-4139. 1

[13] H. S. Hundal, An alternating projection that does not converge in norm, Nonlinear Anal., 57 (2004), 35-61. 2.9

[14] J. S. Jung, Strong convergence of composite iterative methods for equilibrium problems and fixed point problems, Appl. Math. Comput., 213 (2009), 498-505. 1

[15] L. J. Lin, W. Takahashi, A general iterative method for hierarchical variational inequality problems in Hilbert spaces and applications, Positivity, 16 (2012), 429-453. 2.3

[16] Y. Liu, A general iterative method for equilibrium problems and strict pseudo-contractions in Hilbert spaces, Nonlinear Anal., 71 (2009), 4852-4861. 1

[17] S. Plubtieng, R. Punpaeng, A general iterative method for equilibrium problems and fixed point problems in Hilbert spaces, J. Math. Anal. Appl., 336 (2007), 455-469. 1

[18] X. Qin, Y. J. Cho, S. M. Kang, Convergence analysis on hybrid projection algorithms for equilibrium problems and variational inequality problems, Math. Model. Anal., 14 (2009), 335-351. 1

[19] W. Takahashi, Nonlinear functional analysis, Fixed point theory and its applications, Yokohama Publishers, Yokohama, (2000). 2.7

[20] S. Takahashi, W. Takahashi, Viscosity approximation methods for equilibrium problems and fixed point problems in Hilbert spaces, J. Math. Anal. Appl., 331 (2007), 506-515. 1

[21] S. Takahashi, W. Takahashi, M. Toyoda, Strong convergence theorems for maximal monotone operators with nonlinear mappings in Hilbert spaces, J. Optim. Theory Appl., 147 (2010), 27-41. 2.2

[22] M. Tian, An application of hybrid steepest descent methods for equilibrium problems and strict pseudocontractions in Hilbert spaces, J. Inequal. Appl., 2011 (2011), 15 pages. 1

[23] M. Tian, L. Liu, Iterative algorithms based on the viscosity approximation method for equilibrium and constrained convex minimization problem, Fixed Point Theory Appl., 2012 (2012), 17 pages. 1 
[24] M. Tian, L. Liu, General iterative methods for equilibrium and constrained convex minimization problem, Optimization, 63 (2014), 1367-1385. 1, 1, 1

[25] H. K. Xu, Averaged mappings and the gradient-projection algorithm, J. Optim. Theory Appl., 150 (2001), 360378. 2.4

[26] H. K. Xu, Viscosity approximation methods for nonexpansive mappings, J. Math. Anal. Appl., 298 (2004), 279291. 2.10]

[27] H. K. Xu, Averaged mappings and the gradient-projection algorithm, J. Optim. Theory Appl., 150 (2011), 360378. 1

[28] I. Yamada, The hybrid steepest descent method for the variational inequality problem over the intersection of fixed point sets of nonexpansive mappings, Inherently parallel algorithms in feasibility and optimization and their applications, Haifa, (2000), Stud. Comput. Math., North-Holland, Amsterdam, 8 (2001), 473-504. 1

[29] Y. H. Yao, R. P. Agarwal, M. Postolache, Y. C. Liou, Algorithms with strong convergence for the split common solution of the feasibility problem and fixed point problem, Fixed Point Theory Appl., 2014 (2014), 14 pages. 4

[30] Z. T. Yu, L. J. Lin, C. S. Chuang, A unified study of the split feasible problems with applications, J. Nonlinear Convex Anal., 15 (2014), 605-622. 1, 1 